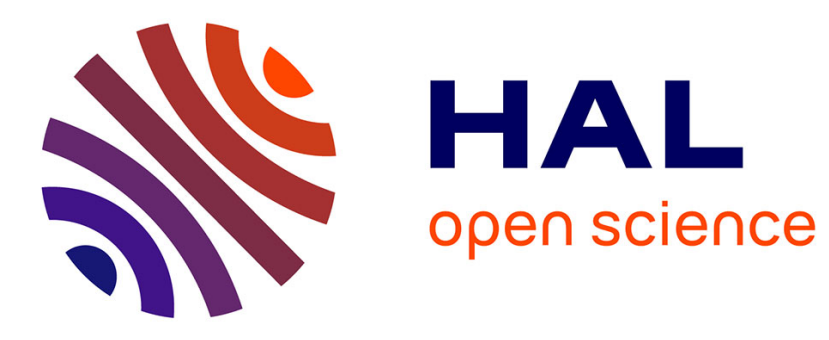

\title{
Validation of a new 2D failure mechanism for the stability analysis of a pressurized tunnel face in a spatially varying sand
}

Guilhem Mollon, Kok Kwang Phoon, Daniel Dias, Abdul-Hamid Soubra

\section{To cite this version:}

Guilhem Mollon, Kok Kwang Phoon, Daniel Dias, Abdul-Hamid Soubra. Validation of a new 2D failure mechanism for the stability analysis of a pressurized tunnel face in a spatially varying sand. Journal of Engineering Mechanics - ASCE, 2011, 137 (1), pp.8-21. 10.1061/(ASCE)EM.1943-7889.0000196 . hal-01007418

\section{HAL Id: hal-01007418 https://hal.science/hal-01007418}

Submitted on 7 Nov 2018

HAL is a multi-disciplinary open access archive for the deposit and dissemination of scientific research documents, whether they are published or not. The documents may come from teaching and research institutions in France or abroad, or from public or private research centers.
L'archive ouverte pluridisciplinaire HAL, est destinée au dépôt et à la diffusion de documents scientifiques de niveau recherche, publiés ou non, émanant des établissements d'enseignement et de recherche français ou étrangers, des laboratoires publics ou privés. 


\title{
Validation of a New 2D Failure Mechanism for the Stability Analysis of a Pressurized Tunnel Face in a Spatially Varying Sand
}

\author{
Guilhem Mollon ${ }^{1}$, Kok Kwang Phoon ${ }^{2}$, Daniel Dias ${ }^{3}$ and Abdul-Hamid Soubra ${ }^{4}$
}

\begin{abstract}
A new two-dimensional (2D) limit analysis failure mechanism is presented for the determination of the critical collapse pressure of a pressurized tunnel face in the case of a soil exhibiting spatial variability in its shear strength parameters. The proposed failure mechanism is a rotational rigid block mechanism. It is constructed in such a manner to respect the normality condition of the limit analysis theory at every point of the velocity discontinuity surfaces taking into account the spatial variation of the soil angle of internal friction. Thus, the slip surfaces of the failure mechanism are not described by standard curves such as log-spirals. Indeed, they are determined point by point using a spatial discretization technique. Though the proposed mechanism is able to deal with frictional and cohesive soils, the present paper only focuses on sands. The mathematical formulation used for the generation of the failure mechanism is first detailed. The proposed kinematical approach is then presented and validated by comparison with numerical simulations. The present failure mechanism was shown to give results (in terms of critical collapse pressure and shape of the collapse mechanism) that compare reasonably well with the numerical simulations at a significantly cheaper computational cost.
\end{abstract}

Author keywords: Tunnels; Active pressure; Limit analysis; Spatial variability; Local weakness.

\section{Introduction}

Stability is a key design/construction consideration in real shield tunnelling projects. The aim of stability analysis is to ensure safety against soil collapse in front of the tunnel face. This paper focuses on the study of the face stability of circular tunnels driven by pressurized shields in the case of a frictional soil. This study requires the determination of the minimal pressure (air, slurry, or earth) required to prevent the collapse of the tunnel face.

The stability analysis of a pressurized tunnel face has been investigated by several writers in the literature. Some writers have performed experimental tests (Chambon and Corté 1994; Takano et al. 2006). Others (Horn 1961; Leca and Dormieux 1990; Eisenstein and Ezzeldine 1994; Anagnostou and Kovari 1996; Broere 1998; Augarde et al. 2003; Klar et al. 2007) have studied the problem using analytical or numerical approaches. More recently, the face stability analysis was investigated by: (1) Mollon et al.

${ }^{1}$ Ph.D. Student, INSA Lyon, Université de Lyon, LGCIE Site Coulomb 3, Géotechnique, Bât. J.C.A. Coulomb, Domaine scientifique de la Doua, 69621 Villeurbanne cedex, France (corresponding author). E-mail: Guilhem.Mollon@insa-lyon.fr

${ }^{2}$ Professor, Dept. of Civil Engineering, National Univ. of Singapore, Singapore. E-mail: cvepkk@nus.edu.sg

${ }^{3}$ Associate Professor, INSA Lyon, Université de Lyon, LGCIE Site Coulomb 3, Géotechnique, Bât. J.C.A. Coulomb, Domaine scientifique de la Doua, 69621 Villeurbanne cedex, France. E-mail: Daniel.Dias@insalyon.fr

${ }^{4}$ Professor, Institut de Recherche en Génie Civil et Mécanique, Univ. of Nantes, UMR CNRS 6183, Bd. de l'université, BP 152, 44603 SaintNazaire cedex, France. E-mail: Abed.Soubra@univ-nantes.fr (2009b, 2010) using the kinematical approach in limit analysis and (2) Mollon et al. (2009a) using numerical simulations as described below.

Mollon et al. (2009b) suggested an analytical threedimensional (3D) multiblock failure mechanism composed of several translational conical blocks, which improved the classical solutions by Leca and Dormieux (1990) but presented the same shortcoming (i.e., the mechanism was not able to deal with the entire circular tunnel face, but only with a vertical inscribed ellipse). This shortcoming was solved in Mollon et al. (2010), in which a new multiblock translational mechanism was presented which intersected the whole circular face. The construction of the slip surfaces of the different blocks was made point by point using a spatial discretization technique since no simple geometrical shape was able to intersect the whole tunnel face. Another approach was also proposed by Mollon et al. (2009a) for the computation of the tunnel collapse pressure. These writers conducted 3D numerical simulations using the finite difference code FLAC3D (Fast Lagrangian Analysis of Continua) (ITASCA Consulting Group 1993).

None of the above papers is related to spatially heterogeneous soils. Hence, the aim of this paper is to establish a suitable deterministic model that is able to deal with the spatial variation of the soil shear strength properties.

Fig. 1 presents the geometry of the problem considered in this paper. This study focuses on pressurized shields using compressed air as the retaining fluid. As a result, the applied face pressure $\sigma_{t}$ is uniform. If this pressure drops below a critical value (critical collapse pressure $\sigma_{c}$ ), the soil mass abutting the tunnel face can collapse into the tunnel. In the case of a frictional 


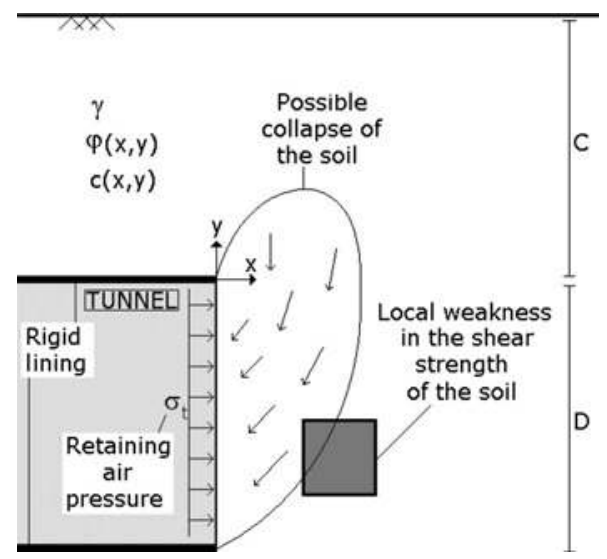

Fig. 1. Problem definition-notations

soil, the arch effect can prevent this failure from reaching the ground surface, especially if the cover depth $C$ is large enough with respect to the diameter $D$ of the tunnel.

This paper proposes a new failure mechanism based on the kinematic theorem of limit analysis for the computation of the critical collapse pressure $\sigma_{c}$ of a spatially varying soil. This mechanism presents two main advantages for downstream stochastic analysis of the tunnel face: (1) it is able to deal with spatial variations of the soil shear strength parameters and (2) it is much less time-consuming than common numerical methods such as the finite-element method (FEM) or the finite difference method (FDM). However, this model has to be validated before using it in an extensive Monte Carlo simulation. This validation is done here by introducing some artificial weaknesses (called "pixels") of several sizes and shapes systematically at several locations in the soil mass. The impact of these weak pixels on the critical collapse pressure is studied. The same cases are treated with the commercial numerical software FLAC3D (although a two-dimensional problem is involved in the present paper) in order to check that the proposed mechanism correctly accounts for these local shear strength weaknesses. The proposed mechanism can be applied to a general $c-\varphi$ soil, but only analysis for purely frictional soils is presented herein.

It should be emphasized here that the present work is undertaken as part of a broader objective to study the impact of a spatially varying soil on the value of the critical collapse pressure $\sigma_{c}$. Realizations of spatially varying soil can be generated quite readily using existing algorithms such as Karhunen-Loeve method and a Monte Carlo sampling method (Phoon et al. 2005). Though reliable, this method requires a large number of calls of the deterministic model, which can be very time-consuming if one uses common numerical methods such as FEM or FDM. For instance, the numerical methods by Augarde et al. (2003), Ukrichton et al. (2003), Hjiaj et al. (2005), Yamamoto et al. (2009), and Abbo et al. (2009) which successfully combined the FEM and the limit analysis theory for the study of stability problems, both in undrained clays and in sands, are promising and can be used in the framework of a spatially varying soil. Notice, however, that these methods are currently not quite practical for extensive Monte-Carlo simulations because of the time cost of each call of the deterministic model. This time cost explains why the collapse mechanism presented in this paper is preferred for the stochastic analysis of pressurized tunnels.

The paper is organized as follows: first a description of the upper-bound approach in limit analysis and the proposed collapse mechanism is presented. This is followed by the presentation of the numerical model using FLAC3D. Finally, the analytical and numerical results obtained in both cases of homogeneous and heterogeneous sands are presented and discussed.

\section{Upper-Bound Theorem in Limit Analysis}

The kinematic theorem in limit analysis is based on the work equation which states that the rate of external forces is equal to the rate of internal energy dissipation for a kinematically admissible velocity field respecting the flow rule and the velocity boundary conditions. For frictional soils, the validity of the upperbound approach has been widely discussed in literature (Chen and Liu 1990; Drescher and Detournay 1993; Soubra and Regenass 2000; Kumar 2004, among others). The assumption of an associated flow rule for frictional soils is at the center of these discussions, because it is widely known from experimental tests that the dilation angle of sands is generally much smaller than the friction angle. However, this assumption is made in the framework of the kinematical theorem of limit analysis, since only for an associated material can the upper-bound theorem be proven true. For an associated flow rule material (for which the velocity characteristics, i.e., the velocity discontinuities in a collapse mechanism, coincide with the stress characteristics often called "slip lines"), the angle between the slip line and the velocity vector should be equal to the soil angle of internal friction. In such a case, the plastic strain increment is normal to the failure criterion line in the Mohr-Coulomb plane (and thus to the stress vector), and the stress vector at the velocity discontinuity has subsequently no dissipative effect (Chen and Liu 1990; Drescher and Detournay 1993). The only energy dissipation in the system is related to cohesion, and the stresses in the soil mass are irrelevant to the solution. In the general case of nonassociated flow rule (i.e., $\psi$ $\neq \varphi$ ), the velocity characteristics do not coincide with the stress characteristics. The stresses are no longer normal to the strain increment, and therefore, they have a dissipating effect. In this case, two approximate (not rigourous) kinematic analyses have been carried out in literature using the following expressions for the equivalent shear strength parameters $\left(c^{*}, \varphi^{*}\right)$ along the velocity characteristics as given by Davis (1968):

$$
\begin{gathered}
c^{*}=c \cdot \frac{\cos \psi \cdot \cos \varphi}{1-\sin \psi \cdot \sin \varphi} \\
\tan \varphi^{*}=\frac{\cos \psi \cdot \sin \varphi}{1-\sin \psi \cdot \sin \varphi}
\end{gathered}
$$

The two kinematic analyses may be described as follows:

- For problems that do not require the determination of the stress distribution along the velocity discontinuity surfaces to be solved by simple statics (such as the case of a translational multiblock failure mechanism) and for which there is an equivalence between the force equilibrium equations and the energy balance equation, Drescher and Detournay (1993) have shown that an approximate solution may be obtained by assuming a fictitous $\left(c^{*}, \varphi^{*}\right)$ soil with an associated flow rule $\psi^{*}=\varphi^{*}$. This is because in these cases, the energy balance equation can be interpreted as an expression of the virtual rate of work principle and thus, an "apparent" energy balance equation can be used for the case of nonassociativeness by selection of a virtual velocity field that is not constrained by the flow rule. Since the limit load does not depend on the 
orientation of the velocity jump, a "fictituous" orientation and thus a fictituous flow rule $\left(\psi^{*}=\varphi^{*}\right)$ has been selected by Drescher and Detournay (1993) for which the specific energy dissipation is independent of the normal stress distribution.

- For problems that do require the determination of the stress distribution along the velocity discontinuity surfaces to be solved by simple statics, Kumar (2004) have made an a priori assumption concerning this distribution using the results of the classical method of slices by Fellenius (1936) and Bishop (1955).

However, one should note that none of these two techniques is able to provide rigorous bounds of the critical load of a system. The assumption of an associated flow rule leads to clear results and clear limitations in the mathematical sense. The key task is to evaluate its limitations carefully with respect to the nonassociated flow rule nature of frictional soils. Finally, one should note that the present paper is not focusing on the exact determination of the critical collapse pressure of a tunnel face, but it deals with the effect of the spatial distribution of the friction angle in the soil when compared to the classical case of a uniform friction angle throughout the soil mass. This specific point will be closely investigated in the next sections, by comparing the analytical results to the ones obtained by a numerical model with associated and nonassociated flow rules.

\section{Collapse Mechanism}

The limit analysis failure mechanism has to provide a correct approximation of the critical collapse pressure. It implies that the failure patterns of the mechanism should not be too different from the physical failures that can be observed during real tunnel face collapses.

Several writers undertook studies of tunnel face collapse on reduced models in frictional soils. Chambon and Corté (1994) conducted their model tests in centrifuge, while the tests by Takano et al. (2006) were undertaken under $1 \mathrm{~g}$. These writers reported that the failure pattern can be modeled by a rotational rigid-block failure mechanism and that the slip surfaces can be approximated by logarithmic spirals. This is in good agreement with the theory of limit analysis which states that a rotational failure in a frictional soil leads to a log-spiral slip surface in the case of a homogeneous soil (i.e., a soil with a constant value of the angle of internal friction). Notice that, to our best knowledge, the mechanism in the presence of spatial variations of the soil shear strength parameters has not been studied yet. Therefore, there is a need to suggest a new failure mechanism that is able to take account of the spatial variations of the soil shear strength properties correctly. This problem is being addressed by the proposed two-dimensional (2D) mechanism described below. As will be seen later, the slip surfaces of the rotational mechanism will not be described by log-spirals in the case of a spatially varying soil but rather by non standard curves that will be searched for point by point using a spatial discretization technique. The assumptions adopted in the analysis are listed below:

- Only the case of a cohesionless soil with an associated flow rule is considered herein.

- For the sake of simplicity, it will be assumed that the failure mechanism never outcrops, which is always true in practice as long as $C>D$ where $C$ and $D=$ tunnel cover and diameter, respectively.

- The 2D failure mechanism intersects the tunnel face in two points: A and B as shown in Fig. 2. Points A and B are intro-

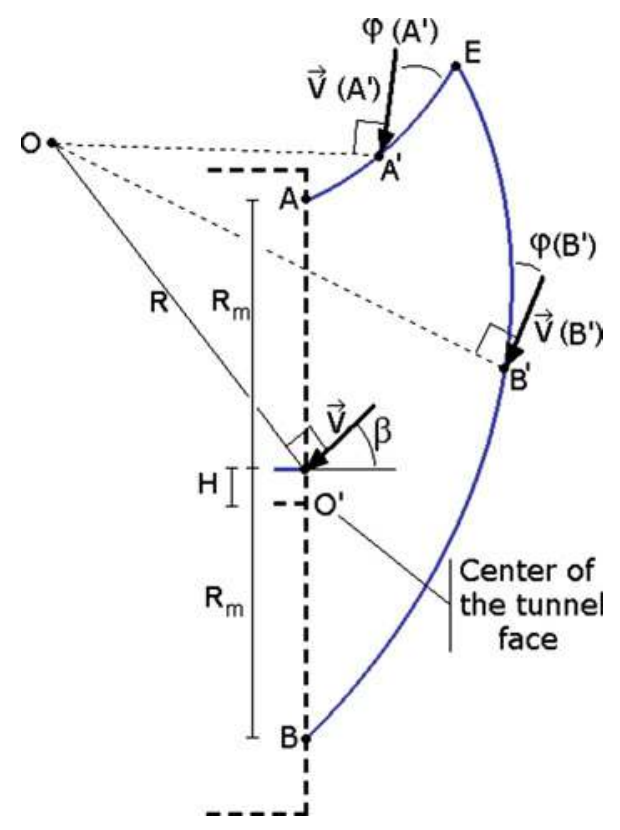

Fig. 2. Normality condition and geometric parameters $\left(R, \beta, H, R_{m}\right)$ used in the rotational failure mechanism

duced here to cater to the possibility of local face collapse in the presence of strength heterogeneities.

- A rotational rigid block failure mechanism is assumed, $O$ being the rotation center.

The mechanism is described by four geometrical parameters as illustrated in Fig. 2. $R$ and $\beta$ are related to the position of the center $O$ of rotation, and $H$ and $R_{m}$ are related to the position of the two points, $\mathrm{A}$ and $\mathrm{B}$, where the mechanism intersects the tunnel face; $O^{\prime}$ being the center of the tunnel face. In a homogeneous soil, the parameters $H$ and $R_{m}$ would be inapplicable because the mechanism would always intersect the whole tunnel face.

The failure mechanism should be kinematically admissible, which implies that the normality condition must be enforced at all points of the velocity discontinuity surfaces taking into account the spatial variation of the soil friction angle. The normality condition requires that the slip surface always makes an angle $\varphi$ with the velocity vector $\mathbf{V}$. If the friction angle is spatially varying, then the slip surface at a point with coordinates $(x, y)$ should be searched for in such a manner to make an angle $\varphi(x, y)$ with the prescribed direction of the velocity vector $\mathbf{V}(x, y)$ at that point (note that $\mathbf{V}(x, y)$ is normal to the corresponding radius), where $\varphi(x, y)$ and $\mathbf{V}(x, y)$ are the spatial distributions of the friction angle and velocity in the soil mass. As shown in Fig. 2, this geometrical condition implies that the slip surfaces emerging from $\mathrm{A}$ and $\mathrm{B}$, respectively, are concave with respect to point $\mathrm{O}$, and that these two lines will eventually meet in a point $E$ which is the extremity of the mechanism. For a homogeneous frictional soil (i.e., with a constant $\varphi$ value throughout the soil mass), the normality condition would lead (if the velocity field is a rigidblock rotation about point $\mathrm{O}$ ) to a rigid block delimited by two logarithmic spirals, coming from points A and B, respectively, and terminating at point $\mathrm{E}$ which constitutes the extremity of the moving block. Such a mechanism would be easy to define analytically, but would not be able to deal with a spatial variation of the friction angle of the soil, i.e., $\varphi(x, y)$.

To address the process of generation of the failure mechanism in the case of a spatially varying soil, an angular discretization 


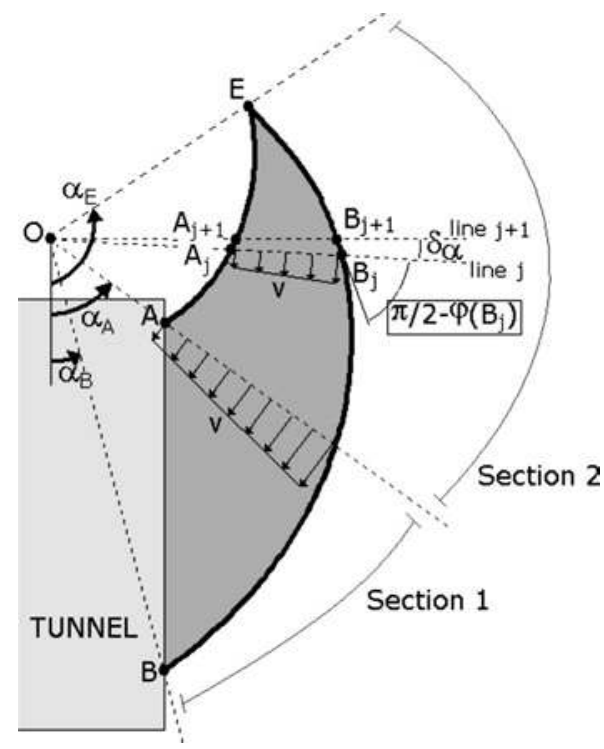

Fig. 3. Principle of generation of the upper and lower slip lines of the failure mechanism

scheme for the two curves emerging from A and B (Fig. 3) was adopted. The generation process uses several radial lines meeting at the point of rotation, $O$. These lines are defined by an index $j$, with $j=0$ corresponding to the first line $O B$. This set of sweeping lines with a common origin $O$ terminates at line $O E$ (unknown a priori). The angle $\delta_{\alpha}$ between two successive radial lines is a user-specified constant. The failure mechanism is divided into two sections. As shown in Fig. 3, Section 1 includes only the lower slip line, and Section 2 includes both the upper and the lower slip lines. The generation process aims at defining a collection of points belonging to the two slip lines. Each point belonging to line $j+1$ is defined from the previous point belonging to line $j$, the whole process starting from points $A$ and $B$ for the upper and lower slip lines, respectively. For example, point $B_{j+1}$ can be deduced from point $B_{j}$ using the two following conditions (the same procedure is applied to deduce $A_{j+1}$ from $A_{j}$ ):

- $B_{j+1}$ belongs to line $j+1$; and

- The straight segment $B_{j} B_{j+1}$ makes an angle $\varphi\left(B_{j}\right)$ with the velocity vector $\mathbf{v}\left(B_{j}\right)$ at point $B_{j}$ (note that the velocity vector is normal to line $j$ ).

As a conclusion, for a spatially varying soil, the friction angle $\varphi$ to be considered at a given point $B_{j}$ of a velocity discontinuity surface is the local value of the friction angle at this point, called $\varphi\left(B_{j}\right)$. It is then straightforward to generate all the points $B_{j}$, from $B$ until the end of Section 1. For Section 2, the same method is also used to generate points $A_{j+1}$ and $B_{j+1}$ from points $A_{j}$ and $B_{j}$, respectively. The upper and lower slip lines are generated until they cross at point $E$. With this generation process, the mechanism is constrained to respect the normality condition at each point $A_{j}$ and $B_{j}$ of its contour. If the soil was homogeneous, the two slip lines emerging from $A$ and $B$ could be analytically defined as logarithmic spirals, which equations would depend on (1) the position of the center of rotation $O$ and (2) the two points A and B, i.e., the four geometrical parameters of the failure mechanism. Fig. 4 shows a plot of these analytical slip lines in a homogeneous sand with $\varphi=30^{\circ}$ where $C=10 \mathrm{~m}$ and $D=10 \mathrm{~m}$, for a given set of the geometrical parameters of the failure mechanism. The contour points obtained by the discretization process described above are also plotted for two values of the angular dis-

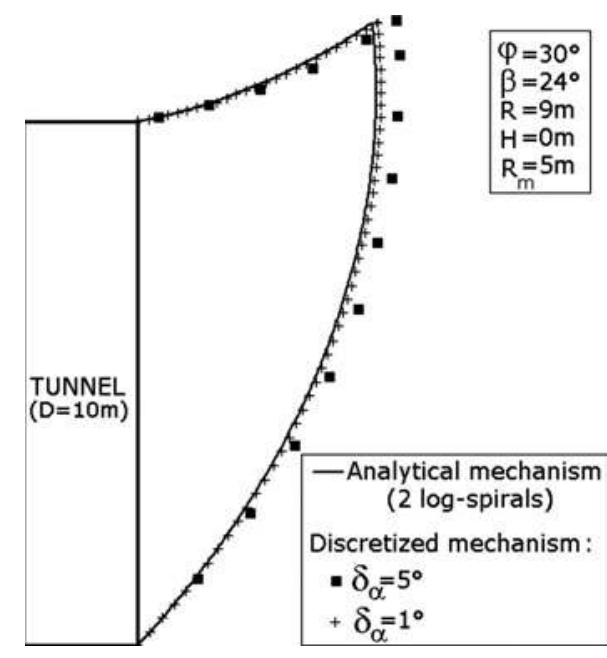

Fig. 4. Effect of the angular discretization parameter $\delta_{\alpha}$ on the contour points of the discretized failure mechanism in the case of a homogeneous soil and comparison with the exact analytical shapes (i.e., log-spirals) of this mechanism

cretization parameter $\delta_{\alpha}$. It appears that this parameter should not be larger than $1^{\circ}$ if one wants to obtain a correct shape of the mechanism, since the contour points for $\delta_{\alpha}=5^{\circ}$ are moving away from the analytical slip lines. One may observe that the principle underlying the discretization process used herein is somewhat similar to the one presented in Mollon et al. (2010). However, the proposed 2D failure mechanism is not a straightforward special case of the 3D mechanism studied previously because the present paper deals with a rotational mechanism which is more complex and more realistic than the translational mechanism presented in Mollon et al. (2010). The writers believe that a 2D study is a necessary first step in the understanding of the behavior of a tunnel face in a spatially varying soil. Generalization of the present rotational mechanism to $3 \mathrm{D}$ may be developed in further studies.

The determination of the collapse pressure corresponding to this mechanism is based on the work equation, which states that the rate of work of the external forces applied to the moving soil mass is equal to the rate of energy dissipation. The applied forces to the moving block are: (1) the weight of the soil composing the moving block; (2) the collapse face pressure; and (3) a possible surcharge loading on the ground surface in the case of outcrop of the mechanism (not considered in the present study). The energy dissipation of the system takes place only at the velocity discontinuity surfaces in the present case of failure mechanism involving rigid block movement. This energy dissipation is proportional to the cohesion (Chen 1975). Since the cohesion is set to zero in the present paper, the energy dissipation is null. Finally, the work equation in the present case of a purely frictional soil with no surcharge loading becomes

$$
\dot{W}_{\gamma}+\dot{W}_{\sigma_{c}}=0
$$

where

$$
\dot{W}_{\sigma_{c}}=\iint_{\Sigma} \boldsymbol{\sigma}_{c} \cdot \mathbf{v} \cdot d \Sigma
$$




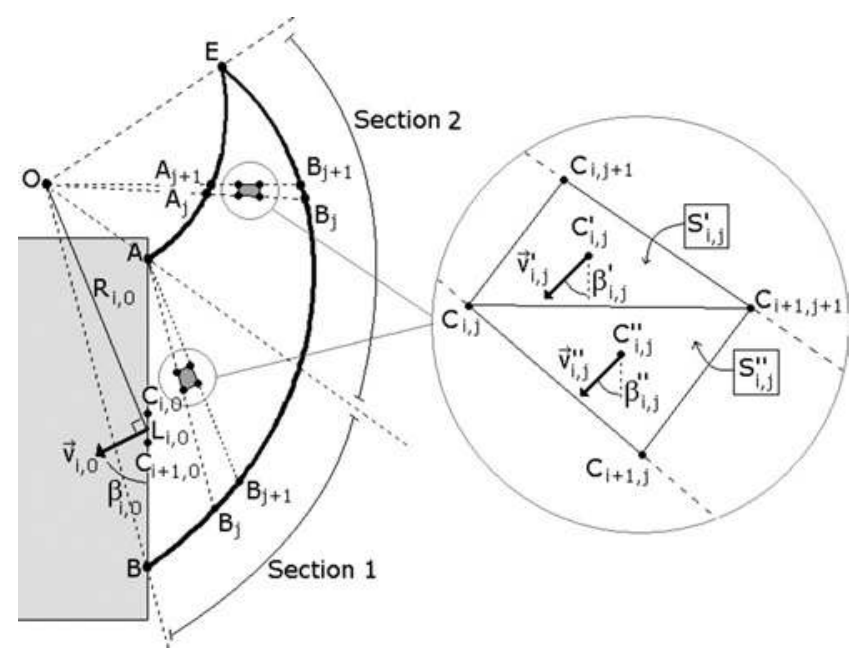

Fig. 5. Principle of the discretization used to solve the work equation

$$
\dot{W}_{\gamma}=\iint_{V} \int \boldsymbol{\gamma} \cdot \mathbf{v} \cdot d V
$$

Computation of Eqs. (4) and (5) was performed numerically by discretizing the volume $V$ of the moving block and the tunnel face surface $\Sigma$ into elementary volumes and surfaces and by summation of the corresponding elementary rates of work. This computation may be explained with the aid of Fig. 5 as follows: the mechanism is first subdivided into several pseudoradial slices. In Section 1, the slices are limited by two successive segments $A B_{j}$ and $A B_{j+1}$, and in Section 2 they are limited by two successive segments $A_{j} B_{j}$ and $A_{j+1} B_{j+1}$. Each segment $j$ [i.e., (1) $A B_{j}$ or $A B$ in Section 1 and (2) $A_{j} B_{j}$ in Section 2] is subdivided into $n_{i}$ segments of equal length. Thus, $n_{i}$ is the number of subdivisions of a slice while $n_{j}$ is the number of slices.

For the computation of the rate of work of the weight of an element $C_{i, j} C_{i+1, j} C_{i, j+1} C_{i+1, j+1}$ (Fig. 5), this element is subdivided into two triangles $C_{i, j} C_{i, j+1} C_{i+1, j+1}$ and $C_{i, j} C_{i+1, j} C_{i+1, j+1}$ of respective barycenters $C_{i, j}^{\prime}$ and $C_{i, j}^{\prime \prime}$ (Fig. 5). Thus, Eqs. (4) and (5) can be written in a discrete way as follows:

$$
\dot{W}_{\sigma_{c}}=-\sum_{i=1}^{n_{i}} \sigma_{c} \cdot L_{i, 0} \cdot \omega \cdot R_{i, 0} \cdot \sin \left(\beta_{i, 0}\right)
$$

$\dot{W}_{\gamma}=\sum_{j=1}^{n_{j}} \sum_{i=1}^{n_{i}}\left[\gamma \cdot S_{i, j}^{\prime} \cdot \omega \cdot R_{i, j}^{\prime} \cdot \cos \left(\beta_{i, j}^{\prime}\right)+\gamma \cdot S_{i, j}^{\prime \prime} \cdot \omega \cdot R_{i, j}^{\prime \prime} \cdot \cos \left(\beta_{i, j}^{\prime \prime}\right)\right]$

where $\omega=$ angular velocity of the moving block; $L_{i, 0}=$ length of an elementary segment $C_{i, 0} C_{i+1,0}$ on the tunnel face; $R_{i, 0}=$ distance between the middle point of segment $C_{i, 0} C_{i+1,0}$ and point $O ; \beta_{i, 0}$ $=$ inclination with the vertical of the velocity vector at the middle point of segment $C_{i, 0} C_{i+1,0} ; S_{i, j}^{\prime}=$ area of triangle $C_{i, j} C_{i, j+1} C_{i+1, j+1}$; $S_{i, j}^{\prime \prime}=$ area of triangle $C_{i, j} C_{i+1, j} C_{i+1, j+1} ; R_{i, j}^{\prime}=$ distance between $C_{i, j}^{\prime}$ and point $O ; R_{i, j}^{\prime \prime}=$ distance between $C_{i, j}^{\prime \prime}$ and point $O ; \beta_{i, j}^{\prime}$ $=$ inclination with the vertical of the velocity vector at point $C_{i, j}^{\prime}$; and $\beta_{i, j}^{\prime \prime}=$ inclination with the vertical of the velocity vector at point $C_{i, j}^{\prime \prime}$. By solving Eq. (3), one obtains the collapse pressure as follows:

$$
\sigma_{c}=\gamma \cdot D \cdot N_{\gamma}
$$

where

$$
N_{\gamma}=\frac{\sum_{j=1}^{n_{j}} \sum_{i=1}^{n_{i}}\left[S_{i, j}^{\prime} \cdot R_{i, j}^{\prime} \cdot \cos \left(\beta_{i, j}^{\prime}\right)+S_{i, j}^{\prime \prime} \cdot R_{i, j}^{\prime \prime} \cdot \cos \left(\beta_{i, j}^{\prime \prime}\right)\right]}{D \cdot \sum_{i=0}^{n_{i}} L_{i, 0} \cdot R_{i, 0} \cdot \sin \left(\beta_{i, 0}\right)}
$$

$N_{\gamma}=$ dimensionless parameter which depends on the size and shape of the mechanism (i.e., on the spatial distribution of the friction angle), and on the four geometrical parameters describing the mechanism (Fig. 1). The collapse pressure defined by Eq. (8) is a rigorous solution in the framework of the kinematical approach of limit analysis. The best (i.e., highest) solution that the mechanism can provide is obtained by maximization of $\sigma_{c}$ with respect to the four geometrical parameters of the failure mechanism. It should be emphasized here that the kinematic theorem allows the determination of an upper bound of the critical load. Since the face pressure is a force resisting collapse, it should be considered as a negative load. The best upper bound of this load is therefore obtained by minimization of $-\sigma_{c}$ (i.e., maximization of $\sigma_{c}$ ). When the soil is homogeneous, then the maximum is unique. This fact has been observed by the writers of this paper and is consistent with the findings of other writers for different stability problems in geotechnical engineering. In this case, the maximization of $\sigma_{c}$ can be performed using a classical optimisation algorithm, such as the optimization tools implemented in Matlab. The maximization procedure has produced in that case two logarithmic spirals curves (results not shown). With a heterogeneous soil, the optimization procedure has produced, as expected, no log-spiral curves and the optimization was no longer possible using a classical optimization procedure because of the possible presence of local maximums for the collapse pressure. As an example, the left parts of Figs. 6(a and b) present two different response surfaces corresponding to two realizations of a random field of the friction angle. These response surfaces are plotted as lines of equal values of the critical collapse pressure in the $(R, \beta)$ plane where the two parameters $R_{m}$ and $H$ are taken equal to 5 and $0 \mathrm{~m}$, respectively (which corresponds to a full-face failure). In these figures, two successive solid lines are separated by $1 \mathrm{kPa}$ and two successive dotted lines are separated by 0.05 $\mathrm{kPa}$. On the right part of Figs. 6(a and b) are plotted the failure patterns corresponding to the global and local maximum values of $\sigma_{c}$. As one can see, several maximums can appear for a given realization of a random field, making it impossible to use traditional optimization methods such as the ones implemented in Matlab.

To obtain the global maximum, an exhaustive search over the full range of the four geometrical parameters was performed. The search for the global maximum was carried out in two steps. First, a coarse "grid" search was done as follows: $15^{\circ} \leq \beta \leq 60^{\circ}$ (step: $2.5^{\circ}$ ); $3 \mathrm{~m} \leq R \leq 20 \mathrm{~m}$ (step: $1 \mathrm{~m}$ ); $-1.5 \mathrm{~m} \leq H \leq 1.5 \mathrm{~m}$ (step: $0.5 \mathrm{~m}$ ); $3.5 \mathrm{~m} \leq R_{m} \leq 5 \mathrm{~m}$ (step: $0.5 \mathrm{~m}$ ). Then, $\sigma_{c}$ was computed for all combinations of the four geometrical parameters and the biggest value of the collapse pressure was conserved. In a second step, the maximum found by using the coarse grid was employed as a starting point for a maximization process using an optimization tool provided by Matlab. This method ensures that the global maximum is found accurately. The two steps of the optimization process require about 4,000 calls of the model and the corresponding computation time was equal to $3 \mathrm{~min}$ (with an angular parameter $\delta_{\alpha}=1^{\circ}$ ) when using a Core2 Quad CPU 2.4GHz PC. More efficient optimization techniques such as the genetic algo- 


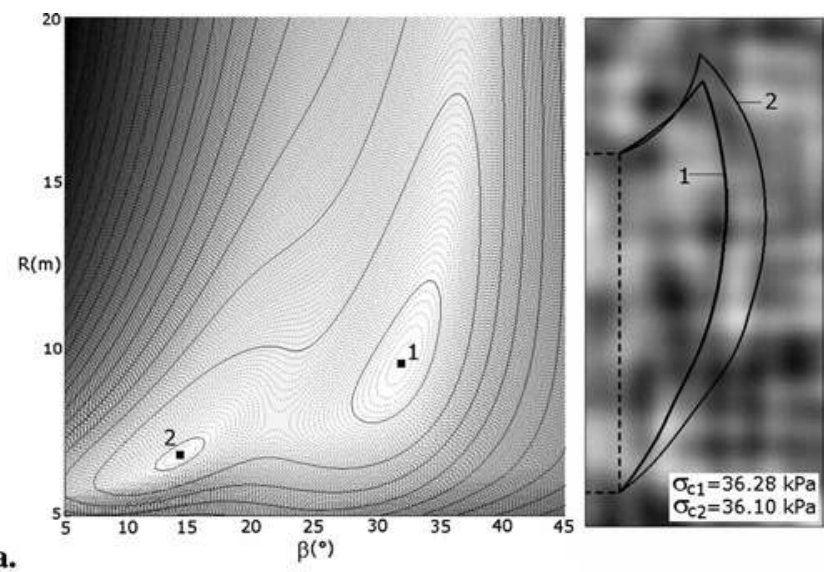

a.

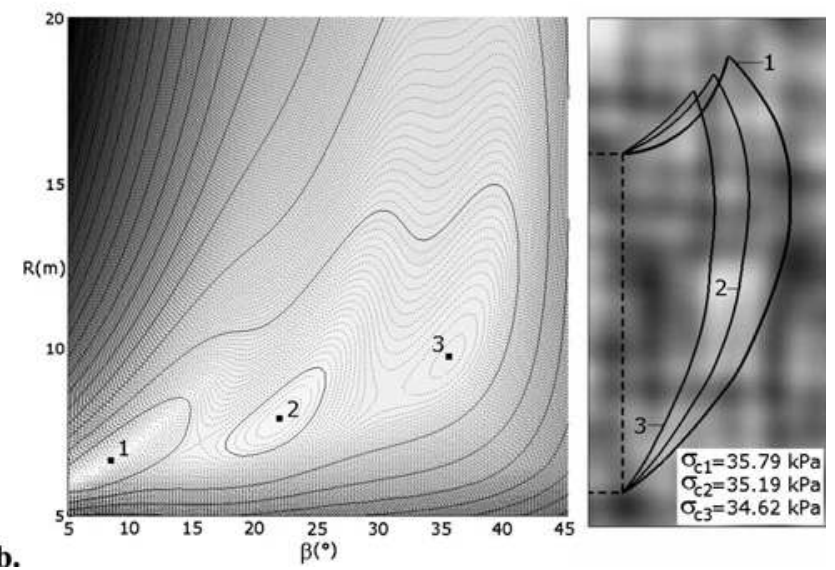

Fig. 6. Response surface of the collapse pressure in the $(\beta, R)$ plane in the case of two random distributions of $\varphi$ (left) and corresponding failure mechanisms in the random soil (dark areas correspond to low $\varphi$ values and bright areas correspond to high $\varphi$ values) corresponding to the different maximums

rithm would be studied in the future in order to reduce the computation time.

\section{Numerical Model}

The numerical simulations presented in this study make use of the 2D numerical model shown in Fig. 7. These simulations are based on FLAC3D software although the problem is two-dimensional.

FLAC3D (Fast Lagrangian Analysis of Continua) (ITASCA Consulting Group 1993) is a commercially available 3D finite difference code in which an explicit Lagrangian calculation scheme and a mixed discretization zoning technique are used. This code includes an internal programming option (FISH) which enables the user to add his own subroutines. In this software, although a static (i.e. nondynamic) mechanical analysis is required, the equations of motion are used. The solution to a static problem is obtained through the damping of a dynamic process by including damping terms that gradually remove the kinetic energy from the system. A key parameter used in the software is the so-called "unbalanced force ratio." It is defined at each calculation step (or cycle) as the average unbalanced mechanical force for all the grid points in the system divided by the average applied mechanical force for all these grid points. The system may be stable (in a steady state of static equilibrium) or unstable (in a

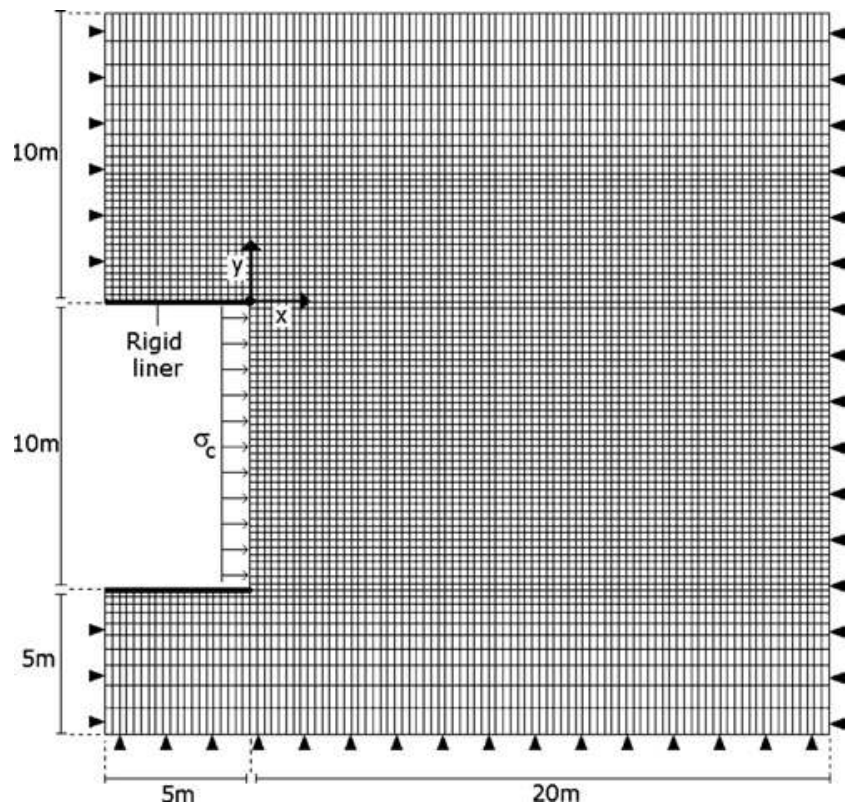

Fig. 7. 2D numerical model used for the determination of the critical collapse pressure using FLAC3D software

steady state of plastic flow). A steady state of static equilibrium is one for which (1) a state of static equilibrium is achieved in the soil-structure system due to given service loads with constant values of the soil displacement (i.e., vanishing values of the velocity) as the number of cycles increases and (2) the unbalanced force ratio becomes smaller than a prescribed tolerance (e.g., $10^{-5}$ as suggested in FLAC3D software) as the number of cycles increases. On the other hand, a steady state of plastic flow is one for which soil failure is achieved. In this case, although the unbalanced force ratio decreases as the number of cycles increases, this ratio does not go to zero but attains a quasi-constant nonvarying value. This value is usually higher than the one corresponding to the steady state of static equilibrium, but can be very small and still lead to infinite displacements, i.e., to failure.

In the model used in this study (Fig. 7), the two vertical and the lower horizontal boundaries are assumed to be fixed in the normal direction. It was shown (not detailed here) that this hypothesis leads to the same results as that for which the different boundaries are fixed on both the horizontal and the vertical directions. The dimensions of the model are $25 \mathrm{~m} \times 25 \mathrm{~m}$. These dimensions were adopted to ensure that the boundaries do not affect the critical collapse pressure (not detailed here). The model is composed of 7,800 zones ("zone" is the FLAC3D terminology for each discretized element) and approximately 16,000 grid points. The tunnel face is divided vertically into 40 zones.

The soil is assigned a perfect elastic-plastic constitutive model based on Mohr-Coulomb criterion with the elastic properties $E$ $=240 \mathrm{MPa}$ and $\nu=0.22$. These elastic properties do not have any significant impact on the critical collapse pressure. For this reason, a very high value of $E$ was chosen because it increases the computation speed. Concerning the soil angle of internal friction and the soil dilatancy angle, their values are given later in the paper. The upper and lower lining of the tunnel are modeled as linear elastic. Their elastic properties are Young's modulus $E$ $=15 \mathrm{GPa}$ and Poisson's ratio $\nu=0.2$. The lining is connected to the soil via interface elements that follow Coulomb's law. The interface is assumed to have a friction angle equal to two thirds of the soil angle of internal friction and no cohesion. Normal stiff- 
ness $K_{n}=10^{11} \mathrm{~Pa} / \mathrm{m}$ and shear stiffness $K_{s}=10^{11} \mathrm{~Pa} / \mathrm{m}$ were assigned to this interface. These parameters are functions of the neighboring elements rigidity (ITASCA Consulting Group 1993). They have almost no influence on the collapse pressure.

The fastest method for the determination of the critical collapse pressure would be a strain-controlled method but it is difficult to apply for stability analysis of tunnels because it assumes that the deflected shape of the tunnel face is known. This shape is not known a priori and any assumption (such as uniform or parabolic deflection) may lead to errors in the determination of the collapse pressure. Thus, a stress-controlled method is used herein. A simple approach would consist of successively applying decreasingly prescribed uniform pressures on the tunnel face until failure occurs. Of course, this requires a significant number of numerical simulations to obtain a satisfactory value of the critical collapse pressure which is very time-consuming. A more rational approach called the bisection method is suggested in this paper and coded in FISH language. It allows the critical collapse pressure of the tunnel face to be determined within an accuracy of 0.1 $\mathrm{kPa}$, and is detailed below.

- The initial lower bracket corresponds to any trial pressure for which the system is unstable. This state corresponds to a nonzero face extrusion velocity (i.e., an infinite displacement) at each point of the tunnel face and means that a steady state of failure or plastic flow is achieved in this case. From a computational point of view, the system is considered as unstable if the tunnel face extrusion continues to increase (the velocity of this extrusion remaining almost constant) after 40,000 computation cycles. Notice that the unbalanced force ratio adopted in this paper is $10^{-7}$ since the value of $10^{-5}$ suggested in FLAC3D software was shown not to give an optimal solution in the present case. The choice of 40,000 for the number of cycles has been determined after several trials: it is large enough to ensure that the system will never be stable if it is still unstable after running these 40,000 cycles.

- The initial upper bracket corresponds to any trial pressure for which the system is stable. This state corresponds to a zero face extrusion velocity (i.e., a constant displacement) at each point of the tunnel face and means that a steady state of static equilibrium is achieved in that case. The system is considered as stable when the unbalanced force ratio drops under $10^{-7}$ before 40,000 computation cycles.

- Next, a new value, midway between the upper and lower brackets, is tested. If the system is stable for this midway value, the upper bracket is replaced by this trial pressure. If the system does not reach equilibrium, the lower bracket is then replaced by the midway value.

- The previous step is repeated until the difference between upper and lower brackets is less than a prescribed tolerance, namely $0.1 \mathrm{kPa}$ in this study. Since the width of the interval is divided by two at each step, a convenient method might be to use a first interval with a width equal to $n$ times $0.1 \mathrm{kPa}, n$ being a power of two.

\section{Validation of the Proposed Mechanism in Homogeneous Sands}

The validity of the proposed mechanism in homogeneous sands is evaluated in this section through a comparison with the numerical model. This comparison is done over the whole range of typical friction angles for sands (i.e., from $30^{\circ}$ to $45^{\circ}$ ), considering a tunnel with $D=10 \mathrm{~m}$ and a soil with $\gamma=18 \mathrm{kN} / \mathrm{m}^{3}$. Two cases

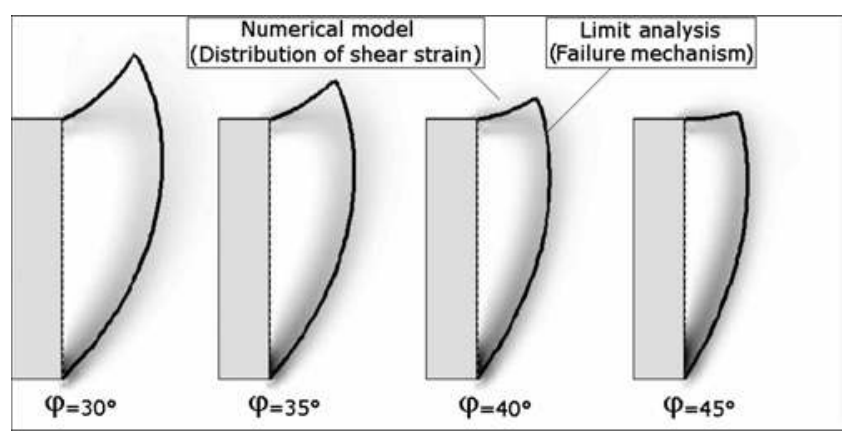

Fig. 8. Slip lines provided by limit analysis and corresponding distributions of plastic shear strain provided by the numerical model in the case of a homogeneous sand

will be studied in the numerical model: the first one with an associated flow rule $(\psi=\varphi)$ and the second one with zero angle of dilatancy $(\psi=0)$. It is believed that the plastic behavior of a real sand is somewhere between these two limit cases. As is well known, numerical models (e.g., FEM, FDM) can easily handle nondilatant behaviors of the soil with a nonassociated flow rule (De Borst 1991; Loukidis and Salgado 2009, among others). The question of whether the numerical problem is even well posed in the case of nonassociated flow is worthy of a brief mention here, since issues related to nonassociated plasticity are not completely resolved in literature.

The results in terms of failure pattern and collapse pressure are given in Figs. 8 and 9. Notice that only the $(\psi=\varphi)$ case is considered in Fig. 8 since the limit analysis assumes an associated flow rule material. The discretization parameter used for the limit analysis mechanism was $\delta_{\alpha}=1^{\circ}$. The computation time was about $3 \mathrm{~min}$ for the limit analysis model and $120 \mathrm{~min}$ for the numerical model, both on a Core2 Quad CPU $2.40 \mathrm{GHz}$. This illustrates the claim that the proposed limit analysis model is much more time efficient and hence, more practical for stochastic simulation. Fig. 8 shows the most critical slip lines provided by limit analysis as well as the corresponding plastic shear strain patterns provided by the numerical model for several values of $\varphi$. There is a reasonably

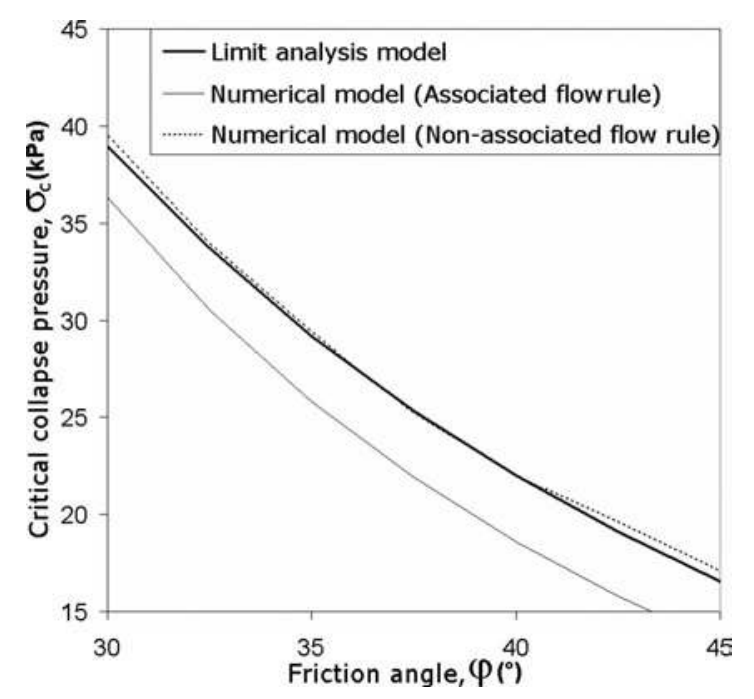

Fig. 9. Comparison between the critical collapse pressures provided by limit analysis and by the numerical model for $\psi=\varphi$ and $\psi=0$ in the case of a homogeneous sand 
good agreement between the two approaches. Fig. 9 presents the $\sigma_{c}$ values provided by limit analysis and by the numerical model (for both $\psi=\varphi$ and $\psi=0$ ) for different $\varphi$ values. The impact of the assumption of associated flow rule clearly appears on this figure. The fact of considering $\psi=\varphi$ instead of $\psi=0$ reduces the critical collapse pressure by $8 \%$ when $\varphi=30^{\circ}$ and by $21 \%$ when $\varphi=45^{\circ}$. The case $\psi=0$ should not be regarded as the real case but as an extreme limit case, because nonzero values of the dilation angle are likely to appear, especially for high friction angles. The analytical curve shows good qualitative agreement in terms of the trend. However, an anomaly should be pointed out. Though the proposed kinematical approach is known to provide a rigorous solution and this solution is expected to be lower (this is because the tunnel pressure resists collapse) than the exact one in the framework of limit analysis, the present mechanism was shown (Fig. 9) to give higher values of the pressure than the numerical model for $\psi=\varphi$ (associated flow rule). This anomaly may be due to the chosen mesh as explained below.

The distributions of the plastic shear strain provided by the numerical model and shown in Fig. 8 appear to be quite spread out when compared to the limit analysis mechanism, which is based on velocity discontinuity surfaces. It should be noted that Chambon and Corté (1994) and Takano et al. (2006) have pointed out a sudden discontinuity in the velocity when they performed their experimental model tests. Hence, the sudden discontinuity used in limit analysis model seems to really reflect the observed sudden collapse phenomenon in sands. The smearing of the shear strain zone given by the numerical model may therefore be interpreted as an effect of the numerical simulation. The obvious cause of smearing is probably the coarseness of the mesh, which governs the velocity gradient. To study this issue, several numerical simulations were realized with locally refined meshes. The refined area was chosen so as to cover all the zones of the mesh with a likely high velocity gradient (i.e., in the vicinity of the velocity discontinuity surfaces obtained from limit analysis), and is illustrated in Fig. 10(a). Different meshes were selected, with progressive local refinement, as presented in Fig. 10(b). The so-called "standard mesh" is similar to the one used earlier in this paper. The critical collapse pressure of the tunnel was calculated for each of these meshes, in the case of an associated flow rule and for two different values of the friction angle $\left(\varphi=30^{\circ}\right.$ and $\varphi$ $=40^{\circ}$ ), using the bisection method detailed earlier. Fig. 11(a) presents the numerical results of this study. It clearly shows that a refined mesh in the zones of high velocity gradients increases the value of the critical collapse pressure with respect to the standard mesh and makes it much more consistent with the results of the limit analysis mechanism (both for $\varphi=30^{\circ}$ and $\varphi=40^{\circ}$ ). It is however necessary to refine the mesh significantly (by a factor 16) for the anomaly pointed out earlier to vanish for both friction angles. Concerning the failure pattern, the local refinement of the mesh makes the distribution of the plastic shear strain much more "concentrated" as shown in Fig. 11(b). A velocity discontinuity is not expected to appear in the numerical model even for an extremely fine mesh, since the concept of discontinuity does not exist in a continuum formulation like the finite difference method. However, the reduction of the width of the shear zone (induced by mesh refinement) increases the gradient of velocity in this zone, and thus makes it closer to the infinite velocity gradient postulated by the analytical model. This is in good agreement with De Borst (1991), who demonstrated that when simulating numerically the shear deformations in a frictional soil submitted to a biaxial-compression test, the width of the shear band was closely related to the coarseness of the mesh. This width was found to
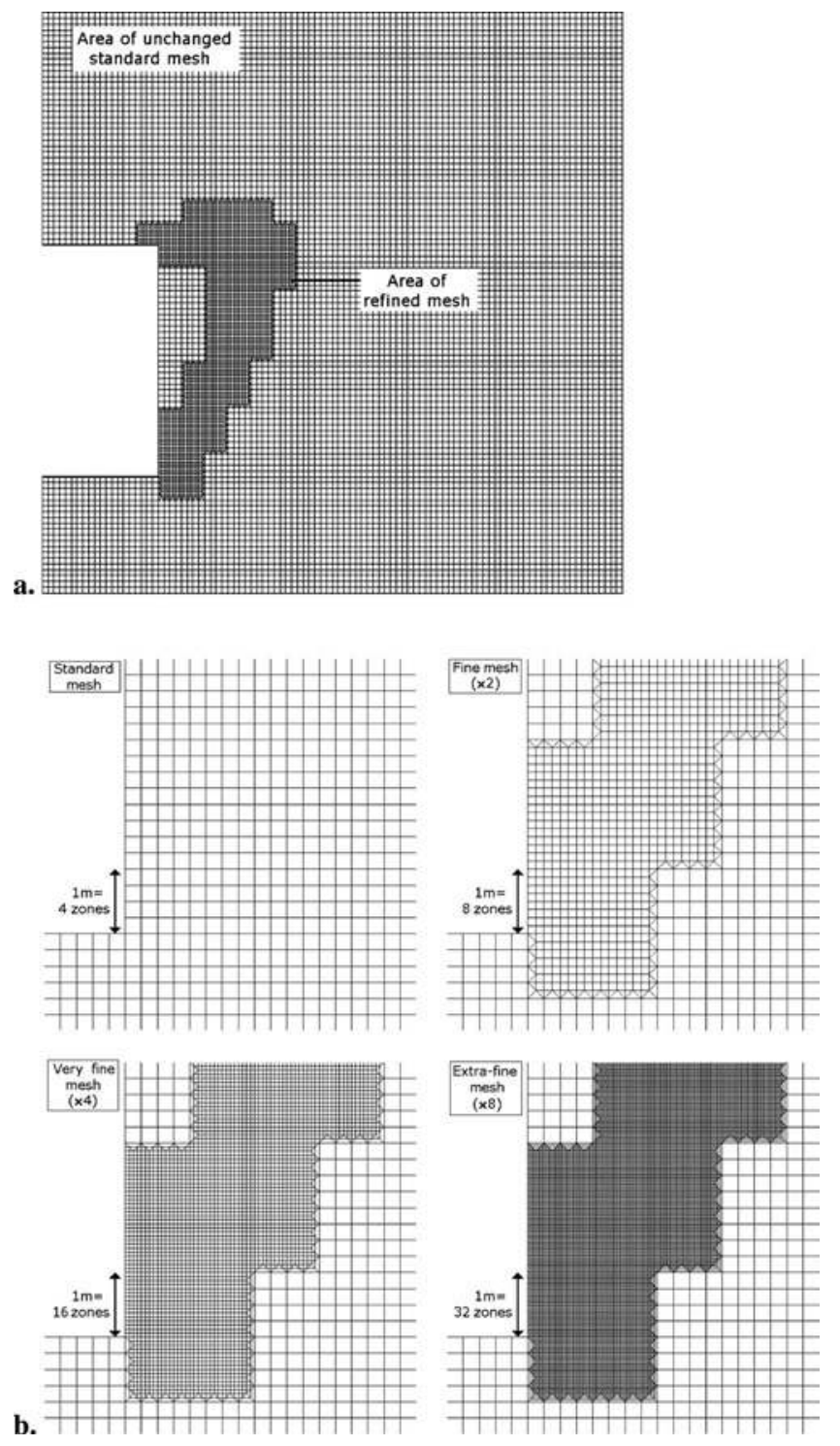

Fig. 10. Locally refined meshes: (a) localization of the refined area; (b) details of the four meshes at the invert of the tunnel face

remain between one and two times the size of a mesh element. It was also found that the coarseness had an impact on the critical pressure, and that a refinement of the mesh led to closer agreement with limit analysis. Thus, the numerical model with a socalled "extra-fine mesh" (i.e., a size of the FLAC3D zone reduced by a factor eight) provides a correct value for the critical collapse pressure and a significant improvement in the shape of the failure, but one should notice that this improvement has a price. The computation time with this model is about 30 times longer than that for the standard mesh, which significantly limits its use in parametric studies or in stochastic simulations. The coarseness of the mesh in the vicinity of high velocity gradients therefore appears to have a significant effect on the results (i.e., on the critical pressure and the corresponding failure pattern) of a numerical simulation. For this reason, two different kinds of meshes will be used from hereon.

- For studies involving a large number of simulations, the standard mesh of the numerical model will be used for the validation of the proposed limit analysis model because it appears to reproduce qualitative trends with reasonable accuracy. When 


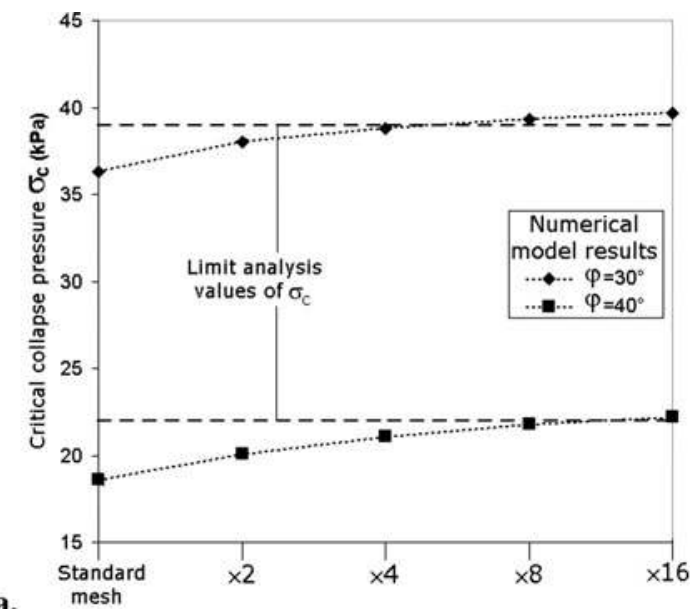

a.

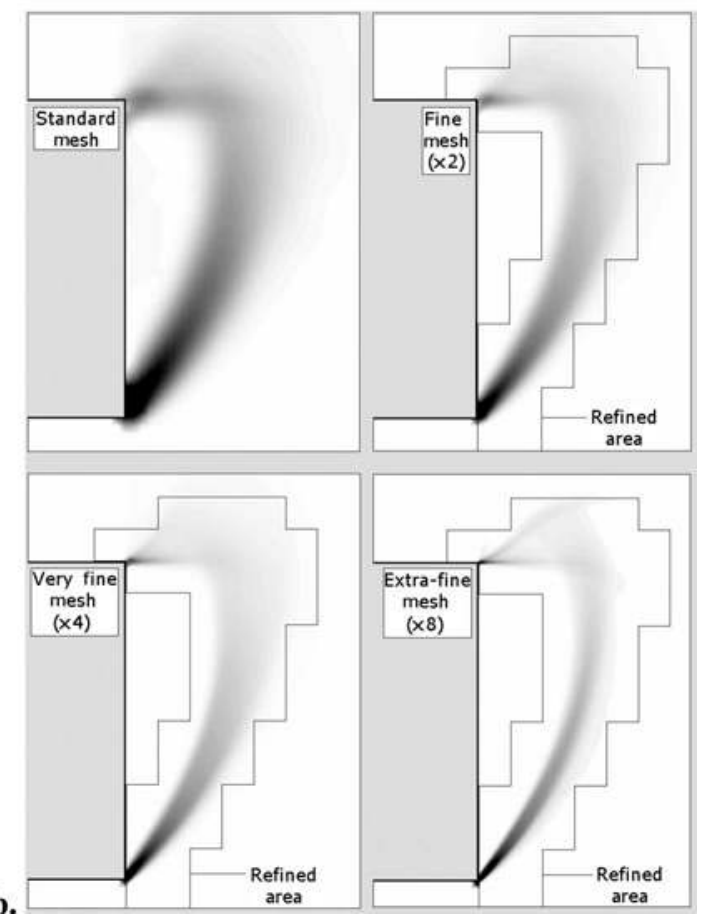

Fig. 11. Effect of mesh local refinement on: (a) critical collapse pressure for $\varphi=30^{\circ}$ and $\varphi=40^{\circ}$; (b) distributions of plastic shear strain for $\varphi=30^{\circ}$

dealing with this standard mesh, all the subsequent results relative to the introduction of a local weakness will be presented in percent increase with respect to the homogeneous case since it is the most relevant way to express the effect of an heterogeneity; the values provided, in the homogeneous case, by both the standard mesh of the numerical model and by the proposed limit analysis method being quite different.

- If more accurate results on the shape of failure and the critical collapse pressure are needed, a locally refined mesh will be used. This mesh will be similar to the "extra-fine" mesh presented earlier, except that the refined area will be adjusted appropriately to cover the expected shear zone of the corresponding simulation.

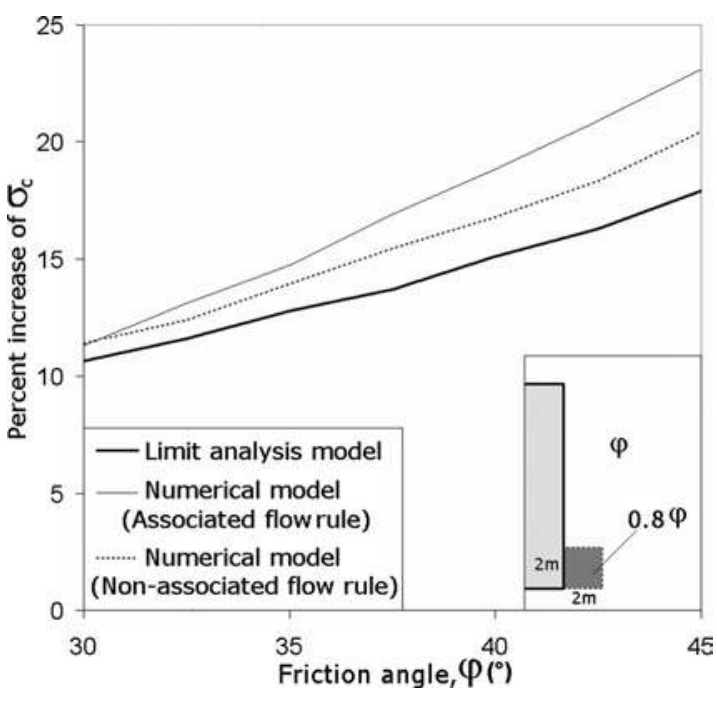

Fig. 12. Percent increase in the critical collapse pressure due to a reduction of $\varphi$ by $20 \%$ in a $2-m$ square pixel at the invert of the tunnel face

\section{Validation of the Proposed Mechanism in Heterogeneous Sands}

The proposed mechanism has been proven to be suitable for homogeneous sands. However, more advanced validations are needed to cover cases of spatially varying sands, which would appear in random field simulations. Two cases were studied: the first one involves a systematic study of the impact of a local weakness in the soil on the critical collapse pressure and the corresponding failure pattern. This study is done by introducing a so-called weak pixel in the soil mass. This pixel may have different locations, sizes and shapes (i.e., square pixel, horizontal layer or vertical layer). The second case considers a real spatially varying soil. Both cases are detailed in the two following sections.

\section{Case of a Local Weakness in the Soil Mass}

For a given simulation, the friction angle remains constant outside of the weak pixel, and is decreased by a given percentage inside the pixel. Each case is dealt with using the proposed mechanism and the numerical model (for both $\psi=\varphi$ and $\psi=0$ ).

\section{Validation in Terms of Collapse Pressures}

Fig. 12 shows the percent increase in the critical collapse pressure as given by limit analysis and by the numerical model in the case where a 2-m square weak pixel located at the invert of the tunnel face is considered in the analysis. As explained before, the results are presented herein in percent increase with respect to the homogeneous case. The friction angle is reduced by $20 \%$ inside the pixel, and the values of $\varphi$ outside the pixel cover the usual range of sands (i.e., $30^{\circ}<\varphi<45^{\circ}$ ). Two limit values of the dilatancy angle are considered in the analysis. The conclusions concerning the results obtained in the present case are very similar to those provided in the homogeneous soil case, i.e., there is a satisfying agreement (the maximal difference is equal to $5 \%$ ) between limit analysis and the numerical model (both for associated and nonassociated rule) when the error induced by the coarseness of the standard mesh of the numerical model is evacuated through the use of a percent increase in $\sigma_{c}$ with respect to the homogeneous case. Moreover, the two numerical curves for associated and non- 
a) Limit analysis

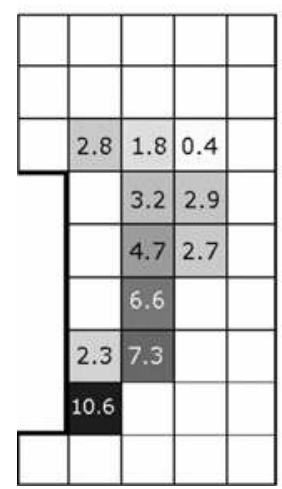

b) Numerical $\operatorname{model}(\psi=\varphi)$

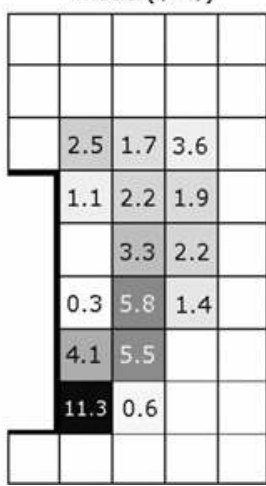

c) Numerical model $(\psi=0)$

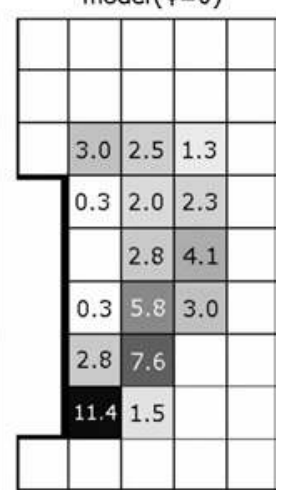

Fig. 13. Percent increase in the critical collapse pressure due to a reduction of $\varphi$ by $20 \%$ in a $2-\mathrm{m}$ square pixel for several locations of this pixel in the soil mass (a white pixel means no impact of this pixel on the face stability)

dilatant soils appear to be quite similar (less than $3 \%$ of difference). This observation (confirmed by the Figs. 13-15 later in this paper) provides an indication of the significance of our study with respect to the associated flow rule limitation highlighted above. As expected, the assumption of associated flow rule can introduce a systematic error in the determination of $\sigma_{c}$ as shown in Fig. 9. However, this assumption affects the relative increase of $\sigma_{c}$ induced by a local weakness in a limited way. In other words, flow rule affects the absolute value of $\sigma_{c}$, but hardly affects the relative change in $\sigma_{c}$, which is the primary goal in this paper.

Fig. 13 shows a more systematic study concerning the impact of the position of a local weakness. In this figure, only a 2-m square pixel with a local reduction of $\varphi$ by $20 \%$ is considered. The friction angle outside of the pixel is equal to $30^{\circ}$ (i.e., the friction angle inside the weak pixel is equal to $24^{\circ}$ ). All the positions of the pixel around the tunnel face are tested and the

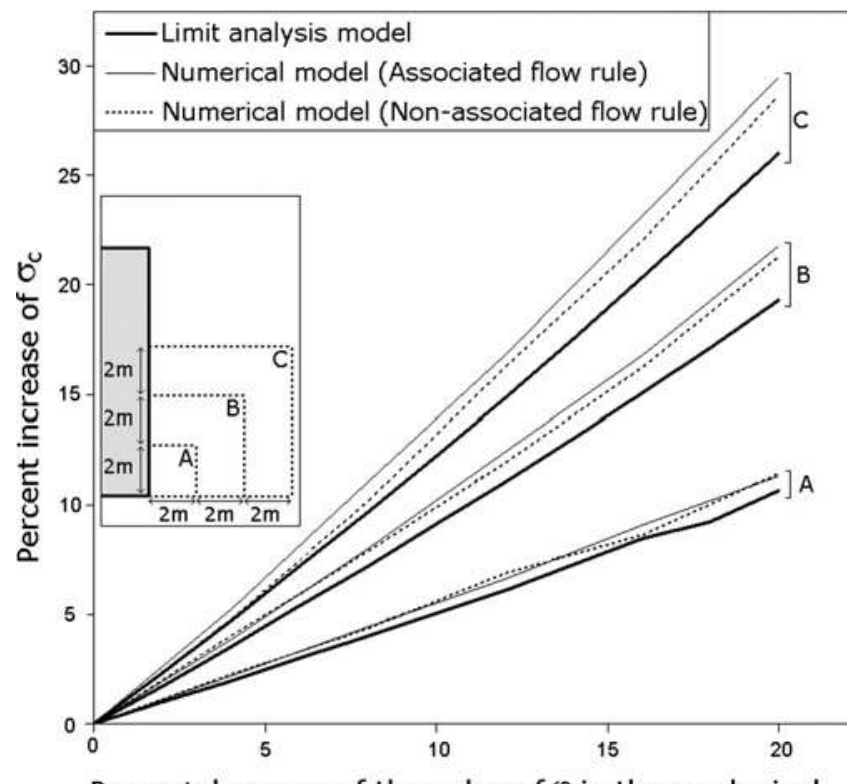

Percent decrease of the value of $\varphi$ in the weak pixel

Fig. 14. Impact of a decrease in the soil friction angle on $\sigma_{c}$ for a pixel located at the invert of the tunnel face for several sizes of the weak pixel
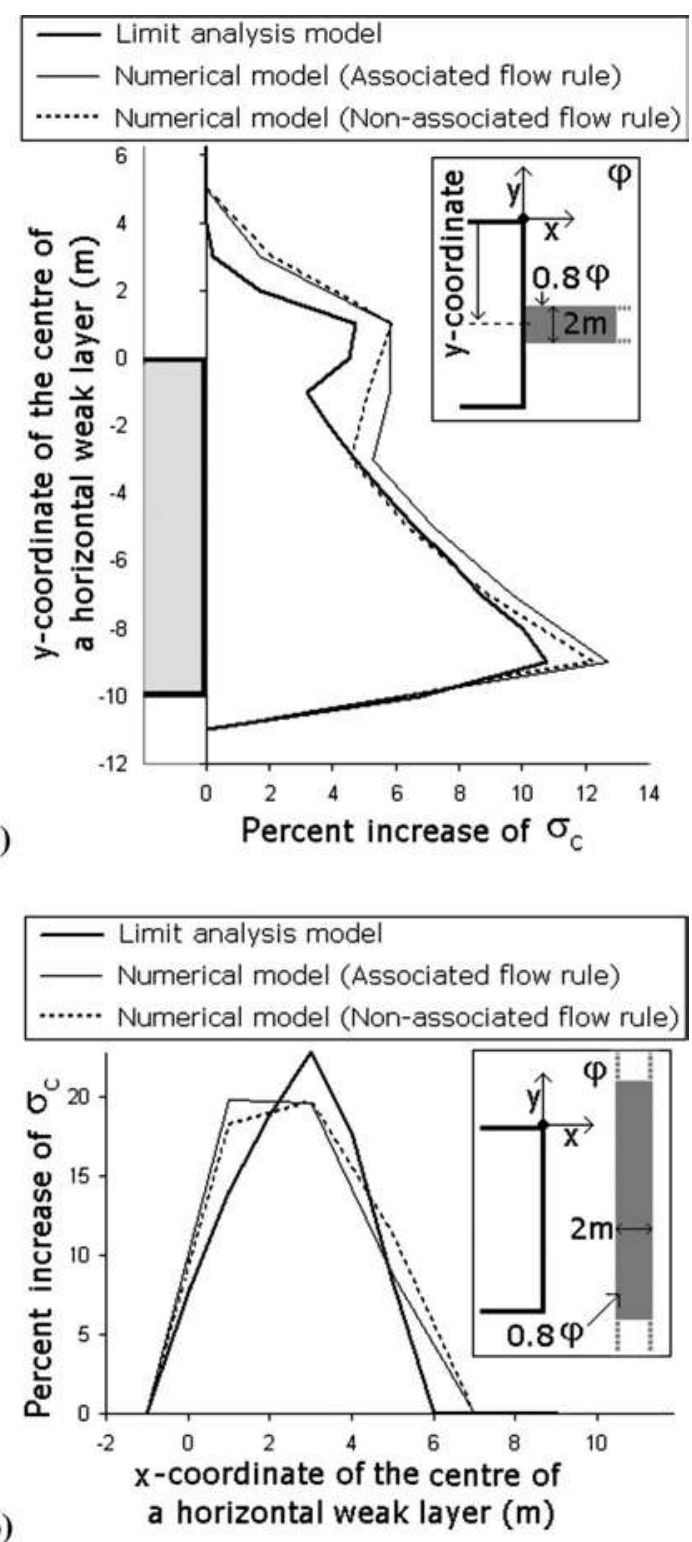

Fig. 15. Percent increase in the critical collapse pressure due to a reduction of $\varphi$ by $20 \%$ in a $2 \mathrm{~m}$-thick weak layer for several locations of this layer in the soil mass in the case of (a) horizontal weak layer; (b) vertical weak layer

critical collapse pressures are computed using both the proposed mechanism and the numerical model (for both $\psi=\varphi$ and $\psi=0$ ). Due to the high number of simulations, the standard mesh was used in the numerical model and the percent increase in $\sigma_{c}$ with respect to the homogeneous case is presented. Fig. 13 therefore presents for each pixel (shade of gray and numerical value) the percent increase in $\sigma_{c}$ caused by a $20 \%$ reduction of $\varphi$ in this pixel. The three models provide quite similar results for the increase of $\sigma_{c}$, which means that: (1) although the dilatancy angle $\psi$ has an effect on the value of $\sigma_{c}$, it has almost no effect on the relative increase of the tunnel collapse pressure in the presence of a local weakness and (2) the proposed failure mechanism gives consistent results with the numerical model when dealing with a local reduction of $\varphi$. The numerical results also show that a local weakness at the invert of the tunnel face has an important impact on the stability, the increase in $\sigma_{c}$ being larger than $10 \%$ with respect to the homogeneous case. A local weakness at the crown 
of the tunnel face does influence the critical collapse pressure but this influence is much smaller than that at the invert. The central part of the tunnel face appears to have a negligible effect on the face stability. The most critical zone for the tunnel face stability is therefore located at the invert of the tunnel face, and it seems to have a significant impact on the critical collapse pressure compared to the other parts of the tunnel face.

The impact of a local decrease of a pixel's friction angle on the tunnel collapse pressure in the case of a pixel located at the tunnel invert is plotted in Fig. 14. It is expressed in terms of a percent increase in the tunnel collapse pressure with respect to the homogeneous case. Three sizes of the weak pixel named Cases A, B, and $\mathrm{C}$ and corresponding, respectively, to 2, 4, and 6-m square pixels are used in the analysis. The friction angle outside of the pixel remains equal to $30^{\circ}$. In the present case, the numerical model makes use of the standard mesh. Once again, the proposed mechanism shows a satisfying agreement with the results of the numerical model, both for associated and nonassociated sands. The critical collapse pressure appears to increase linearly with the local decrease of $\varphi$ for the three sizes of pixels. Moreover, the impact of the local weakness increases with the size of the pixel: a local reduction of $\varphi$ by $20 \%$ leads to an increase in $\sigma_{c}$ by 10.6 , 19.3, and $26.0 \%$ for Cases A, B, and C, respectively.

A similar study was carried out with a weak layer instead of a weak pixel. A horizontal and a vertical layer with a thickness equal to $2 \mathrm{~m}$ were considered in the analysis. A vertical layer is clearly not likely to appear in a real soil (though not impossible), but is used here to study the impact of a local weakness which would be more extended in the vertical direction than in the horizontal one. Both vertical and horizontal weaknesses can appear in an isotropic random field due to fortuitous aggregation and alignment of weak spots in a preferred direction. We expect vertical weakness to be more critical and hence it is included in this study. In Fig. 15(a), the percent increase in $\sigma_{c}$ due to a $2 \mathrm{~m}$-thick horizontal weak layer was plotted with respect to the $y$-coordinate of the center of this weak layer. Similarly, Fig. 15(b) presents the percent increase in $\sigma_{c}$ due to a 2 -m thick vertical weak layer with respect to the $x$-coordinate of the center of this weak layer. The reduction of $\varphi$ in the horizontal and vertical layers is equal to $20 \%$, and the value of $\varphi$ outside the weak layer is $30^{\circ}$. The proposed kinematical approach gives correct results when compared to the numerical model for both cases of a horizontal or a vertical layer, which confirms the observations of the previous paragraph. The most critical horizontal layer is the one located at the invert of the tunnel face, and the corresponding increase in $\sigma_{c}$ is larger than $10 \%$ with respect to the homogeneous case. As expected, a vertical layer has a larger impact on the stability; probably because the shape of failure is mainly vertical (i.e., the slip lines of the failure mechanism are following directions that are not far from the vertical). A $2-\mathrm{m}$ thick vertical layer which center is located between $2 \mathrm{~m}$ and $4 \mathrm{~m}$ behind the tunnel face can increase $\sigma_{c}$ by more than $20 \%$.

\section{Validation in Terms of Failure Patterns}

Only the most relevant cases where the soil heterogeneity may have an effect on the failure pattern are plotted in Fig. 16. Cases (a) and (b) correspond to 4-m square weak pixels located, respectively, at the invert and at the crown of the tunnel, and Cases (c) and (d) correspond to 2-m thick vertical layers located at two different distances from the tunnel face. The reduction of $\varphi$ in the weak pixels or layers remains at $20 \%$ and the friction angle outside the pixels or layers is taken equal to $30^{\circ}$. The failure patterns provided by both the limit analysis failure mechanism and by the

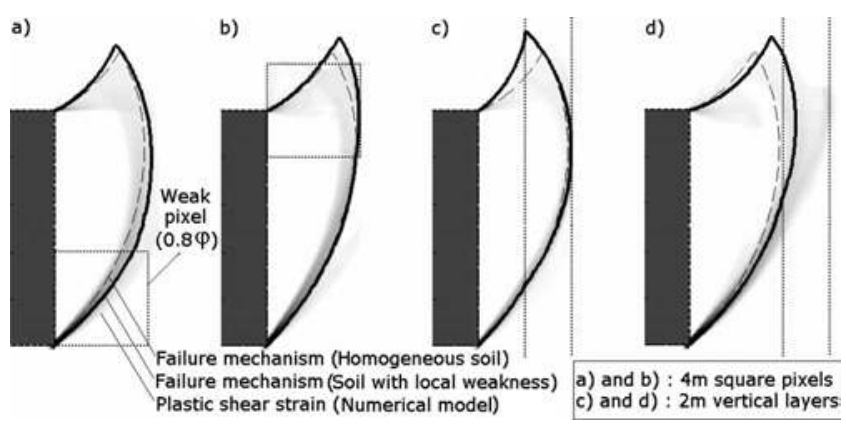

Fig. 16. Failure patterns provided by the proposed failure mechanism and by the numerical model (with an extra fine mesh) for several shapes and positions of the local weakness in the soil mass and comparison with the failure mechanism of a homogeneous soil

numerical model (with the so-called extra-fine mesh) are presented in Fig. 16. They show reasonable agreement. For Case (a), the shape of the failure pattern does not differ significantly from that of the homogeneous case. For Case (b), the mechanism is stretched vertically in the weak pixel located at the crown of the tunnel face. Cases (c) and (d) show that the vertical weak layers can "attract" the failure mechanism much more than the weak square pixels because the lower slip line of the mechanism is aligned in a predominantly vertical direction. Based on this observation, one can postulate that an "inclined" layer at the invert of the tunnel face would probably be the most critical shape for a local weakness, because it would be located in the most critical area (invert of the face) and would "fit" the lower slip line even better than a vertical layer.

\section{Case of a Random Soil}

The validation of the failure mechanism in spatially varying soils is undertaken in this section. Two 2D random fields of the friction angle $\varphi$ were generated using Karhunen-Loeve expansion method (Phoon et al. 2005) and were applied to the limit analysis model and to the numerical model (with the extra-fine mesh). The two fields are lognormal spatial distributions of $\varphi$ with a mean value $\mu_{\log }=30^{\circ}$ and a standard deviation $\sigma_{\log }=3^{\circ}$ (i.e., a coefficient of variation $\mathrm{COV}$ of $10 \%$ ). To generate such a field with the Karhunen-Loeve expansion method, one first has to generate a normal random field with a mean value $\mu_{\text {normal }}$ and a standard deviation $\sigma_{\text {normal }}$. The first two moments of this underlying normal field can be obtained by using the target moments of the lognormal random field $\left(\mu_{\log }\right.$ and $\left.\sigma_{\log }\right)$ as follows:

$$
\begin{gathered}
\sigma_{\text {normal }}=\sqrt{\ln \left(1+\frac{\sigma_{\log }^{2}}{\mu_{\log }^{2}}\right)} \\
\mu_{\text {normal }}=\ln \left(\mu_{\log }\right)+\frac{1}{2} \cdot \sigma_{\text {normal }}^{2}
\end{gathered}
$$

The underlying normal fields are assumed to follow an exponential autocorrelation function, i.e., the coefficient of correlation between two points, A and B, can be obtained by the following expression (Sudret and Der Kiureghian 2000):

$$
\rho(A, B)=\exp \left(-\frac{\left|x_{A}-x_{B}\right|}{L_{x}}-\frac{\left|y_{A}-y_{B}\right|}{L_{y}}\right)
$$

In the present paper, only isotropic random fields (i.e., where the autocorrelation lengths $L_{x}$ and $L_{y}$ are such that $L_{x}=L_{y}=L$ ) are 


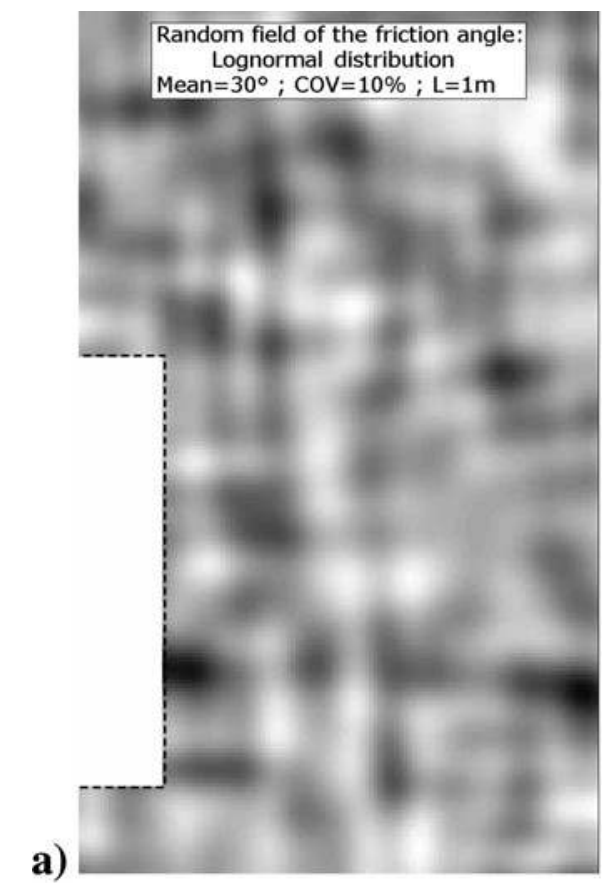

\begin{tabular}{|c|c|c|}
\hline & Limit analysis & Numerical model \\
\hline Homogeneous case & $\sigma_{c}=38.96 \mathrm{kPa}$ & $\sigma_{\mathrm{c}}=39.3 \mathrm{kPa}$ \\
\hline Random field & $\sigma_{\mathrm{c}}=40.85 \mathrm{kPa}$ & $\sigma_{\mathrm{c}}=41.6 \mathrm{kPa}$ \\
\hline
\end{tabular}
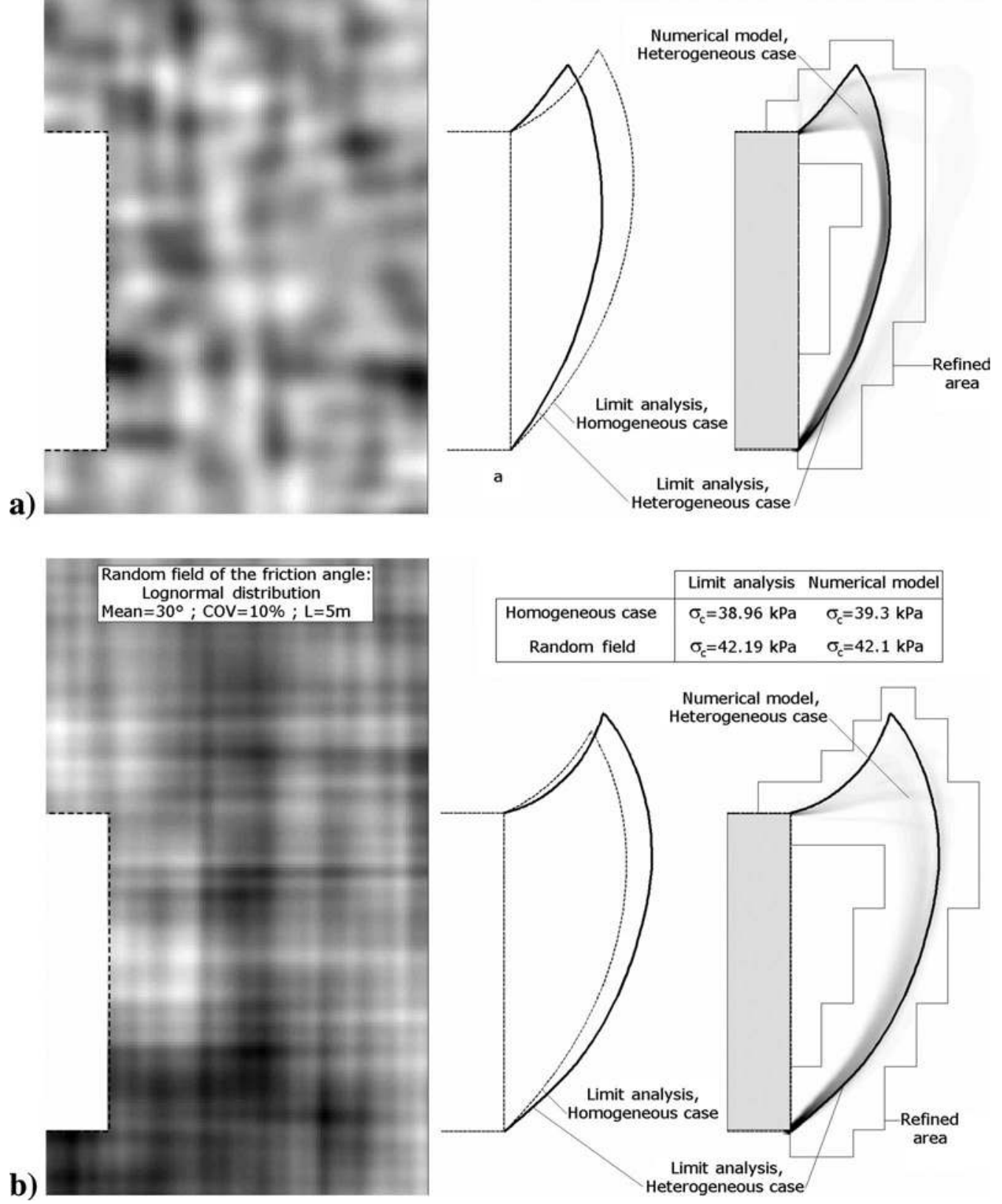

Fig. 17. Random fields of the friction angle for two values of the autocorrelation distance (left), resulting failure patterns provided by the numerical model and by the proposed failure mechanism (right) and comparison with the failure mechanism of a homogeneous soil (center)

considered in the analysis. It can be demonstrated that, as long as the coefficient of variation of the random field remains small (which is the case when dealing with the friction angle), the autocorrelation function of the lognormal field is very similar to the one of the underlying normal field (Sudret and Der Kiureghian 2000). The two lognormal random fields used in the present studies are therefore considered to follow the autocorrelation function given by Eq. (12), with respective autocorrelation lengths of $L$ $=1 \mathrm{~m}$ and $L=5 \mathrm{~m}$.

The left parts of Figs. 17( $a$ and $b$ ) show the two random fields around the tunnel face (the dark areas correspond to low friction angles and the light areas correspond to high friction angles). The numerical model and the proposed limit analysis approach produce comparable results in terms of the collapse pressure. The failure patterns provided by the proposed failure mechanism and by the numerical model for the two random soils are compared on the right part of Figs. 17( $\mathrm{a}$ and $\mathrm{b}$ ). The slip lines appear less "regular" than in the homogeneous case because of the spatial variation of the friction angle and subsequently of the local value of the angle of internal friction that should exist at each point of the slip lines between the slip line and the velocity vector. The failure mechanisms and the corresponding collapse pressures provided by the two models are very similar for both random fields, which imply that the proposed limit analysis approach can be 
used with confidence for downstream stochastic simulations. Field 1 leads to a relatively smaller and vertical failure zone, while Field 2 leads to a more extended failure in the horizontal direction. Such an observation based on only two samples is obviously tentative and will have to be confirmed by an extensive Monte-Carlo simulation. This topic will be the subject of future studies.

Finally, the failure patterns and the corresponding collapse pressures obtained by the proposed failure mechanism in both the homogeneous soil and the spatially varying soil are presented in the central part of Figs. 17( $\mathrm{a}$ and $\mathrm{b}$ ). It appears that there is no direct relationship between the size and shape of the mechanism and the critical collapse pressure for a given sample of the random field.

\section{Conclusions}

This paper aimed at presenting and validating a new 2D failure mechanism for the determination of the critical collapse pressure of a pressurized tunnel face in the case of a sand layer exhibiting several types of weaknesses [(1) a local weakness represented by a weak pixel or a weak layer or (2) a more general case of weakness concerning a random soil represented by an autocorrelation function]. Validation is carried out by comparison with $2 \mathrm{D} \mathrm{nu}-$ merical model using the commercial software FLAC3D. The proposed kinematical approach provides consistent results both in terms of the collapse pressure and the failure pattern when compared to the numerical model, and can therefore be used with confidence in soils modeled as random fields. Moreover, the assumption of associated flow rule used in the analytical model was tested with the numerical model. It appeared that this assumption leads to a systematic underestimation of the critical collapse pressure (from 8 to $21 \%$ for the common values of $\varphi$ in a sand with respect to the limit case $\psi=0$ ), but has very little impact when dealing with the relative change of the critical pressure from a homogeneous to a spatially heterogeneous distribution of $\varphi$.

The systematic study of the impact of local weaknesses (weak pixels or weak layers) on the face stability has demonstrated that the most critical weakness area is the one located at the invert of the tunnel face.

This study has two important ramifications. First, the discretization technique used for the generation of the failure mechanism can also be used to compute the failure load or the safety factor in spatially varying soils relevant to other stability problems in geotechnical engineering, such as footings and slopes. Second, the significant computational savings would bring Monte Carlo simulations of random field problems (basically all stability problems in geotechnical engineering) within reach of the average practitioner using modest computing platforms. Current random finite element methods based on Monte Carlo simulations are too tedious to be applied to routine problems in practice.

\section{Acknowledgments}

This research was carried out during a stay of the first writer in the Department of Civil Engineering at the National University of Singapore from March to September 2009, with the permission and financial support of the LGCIE (Laboratoire de Génie Civil et d'Ingénierie Environementale, INSA Lyon, Université de Lyon, France). These two institutions are gratefully acknowledged.

\section{References}

Abbo, A. J., Wilson, D. W., Lyamin, A. V., and Sloan, S. W. (2009). "Undrained stability of a circular tunnel." Proc., EURO:TUN 2009 Congress, Aedificatio Publishers, Freiburg, Germany, 857-864.

Anagnostou, G., and Kovari, K. (1996). "Face stability conditions with earth-pressure-balanced shields." Tunn. Undergr. Space Technol., 11(2), 165-173.

Augarde, C. E., Lyamin, A. V., and Sloan, S. W. (2003). "Stability of an undrained plane strain heading revisited." Comput. Geotech., 30, 419-430.

Bishop, A. W. (1955). "The use of slip-circle in the stability analysis of slopes." Geotechnique, 5(1), 7-17.

Broere, W. (1998). "Face stability calculation for a slurry shield in heterogeneous soft soils." Proc., World Tunnel Congress 98 on Tunnels and Metropolises, Vol. 1, Balkema, Rotterdam, The Netherlands, 215218.

Chambon, P., and Corté, J. F. (1994). "Shallow tunnels in cohesionless soil: Stability of tunnel face." J. Geotech. Engrg., 120(7), 1148-1165.

Chen, W. F. (1975). Limit analysis and soil plasticity, Elsevier, Amsterdam, The Netherlands.

Chen, W. F., and Liu, X. L. (1990). Limit analysis in soil mechanics, Elsevier Science, Amsterdam, The Netherlands.

Davis, E. H. (1968). "Theories of plasticity and the failure of soil masses." Soil mechanics: Selected topics, I. K. Lee, ed., Butterworth, London, 341-380.

De Borst, R. (1991). "Numerical modelling of bifurcation and localisation in cohesive-frictional materials." Pure Appl. Geophys., 137(4), 367-390.

Drescher, A., and Detournay, E. (1993). "Limit load in translational failure mechanisms for associated and non-associated materials." Geotechnique, 43(3), 443-456.

Eisenstein, A. R., and Ezzeldine, O. (1994). "The role of face pressure for shields with positive ground control." Tunneling and ground conditions, Balkema, Rotterdam, The Netherlands, 557-571.

Fellenius, W. (1936). "Calculation of stability of earth dams." Proc., Transactions 2nd Congress on Large Dams, Vol. 4, International Commission on Large Dams, Washington, D.C.

Hjiaj, M., Lyamin, A. V., and Sloan, S. W. (2005). "Numerical limit analysis solutions for the bearing capacity factor $\mathrm{N}_{\gamma}$." Int. J. Solids Struct., 42, 1681-1704.

Horn, N. (1961). "Horizontaler erddruck auf senkrechte abschlussflächen von tunnelröhren." Landeskonferenz der ungarischen tiefbauindustrie, Deutsche Überarbeitung durch STUVA, Düsseldorf, 7-16.

ITASCA Consulting Group. (1993). FLAC3D: Fast Lagrangian analysis of continua, Minneapolis.

Klar, A., Osman, A. S., and Bolton, M. (2007). "2D and 3D upper bound solutions for tunnel excavation using 'elastic' flow fields." Int. J. Numer. Analyt. Meth. Geomech., 31(12), 1367-1374.

Kumar, J. (2004). "Stability factors for slopes with non-associated flow rule using energy considerations." Int. J. Geomech., 4(4), 264-272.

Leca, E., and Dormieux, L. (1990). "Upper and lower bound solutions for the face stability of shallow circular tunnels in frictional material." Geotechnique, 40(4), 581-606.

Loukidis, D., and Salgado, R. (2009). "Bearing capacity of strip and circular footings in sand using finite elements." Comput. Geotech. 36(5), 871-879.

Mollon, G., Dias, D., and Soubra, A.-H. (2009a). "Probabilistic analysis of circular tunnels in homogeneous soils using response surface methodology." J. Geotech. Geoenviron. Eng., 135(9), 1314-1325.

Mollon, G., Dias, D., and Soubra, A.-H. (2009b). "Probabilistic analysis and design of circular tunnels against face stability." Int. J. Geomech., 9(6), 237-249.

Mollon, G., Dias, D., and Soubra, A.-H. (2010). "Face stability analysis of circular tunnels driven by a pressurized shield." J. Geotech. Geoenviron. Eng., 136(1), 215-229.

Phoon, K. K., Huang, H. W., and Quek, S. T. (2005). "Simulation of 
strongly non-Gaussian processes using Karhunen-Loeve expansion.” Probab. Eng. Mech., 20(2), 188-198.

Soubra, A.-H., and Regenass, P. (2000). "Three-dimensional passive earth pressures by kinematical approach." J. Geotech. Geoenviron. Eng., 126(11), 969-978.

Sudret, B., and Der Kiureghian, A. (2000). "Stochastic finite element methods and reliability, a state of the art." Rep. No. UCB/SEMM2000/08, Dept. of Civil and Environmental Engineering, Univ. of California, Berkeley, Berkeley, Calif.

Takano, D., Otani, J., Nagatani, H., and Mukunoki, T. (2006). “Applica- tion of X-ray CT boundary value problems in geotechnical engineering-Research on tunnel face failure." Proc., Geocongress, ASCE, Reston, Va., 1-6.

Ukrichton, B., Whittle, A. J., and Klangvijit, C. (2003). "Calculations of bearing capacity factor $\mathrm{N}_{\gamma}$ using numerical limit analysis." J. Geotech. Geoenviron. Eng., 129(6), 468-474.

Yamamoto, K., Lyamin, A. V., Wilson, D. W., Abbo, A. J., and Sloan, S. W. (2009). "Limit analysis of shallow tunnels in cohesive-frictional soils." Proc., EURO:TUN 2009 Congress, Aedificatio Publishers, Freiburg, Germany, 857-864. 